\title{
PD-1 and PD-L1 are more highly expressed in high-grade bladder cancer than in low- grade cases: PD-L1 might function as a mediator of stage progression in bladder cancer
}

\author{
Takashi Kawahara', Yukari Ishiguro', Shinji Ohtake', Ikuma Kato², Yusuke Ito ${ }^{1}$, Hiroki Ito ${ }^{1}$, Kazuhide Makiyama', \\ Keiichi Kondo', Yasuhide Miyoshi', Yasushi Yumura', Narihiko Hayashi', Hisashi Hasumi ${ }^{1}$, Kimito Osaka', \\ Kentaro Muraoka ${ }^{1}, K_{0 j i}$ Izumi ${ }^{1}$, Jun-ichi Teranishi ${ }^{1}$, Hiroji Uemura ${ }^{1,3}$, Masahiro Yao ${ }^{1}$ and Noboru Nakaigawa ${ }^{1 *}$ (D)
}

\begin{abstract}
Background: Bladder cancers have been characterized as a tumor group in which the immunological response is relatively well preserved. Programmed death ligand 1 (PD-L1, B7-H1, CD274) has been shown to be expressed in several malignancies, including bladder cancer. However, the clinicopathological impact of this biomarker has not yet been established. In the present study, a quantitative real-time polymerase chain reaction (qPCR) was performed using paired normal and cancerous bladder cancer tissue to investigate PD-1/PD-L1 gene expression.

Methods: We examined the mRNA expression of PD-1/PD-L1 by a qPCR using 58 pairs of normal and cancerous human bladder tissue specimens. We also examined the correlation with the expressions of the STAT1 and NFAT genes, which are thought to be upstream and downstream of the PD-L1 pathway, respectively.

Results: There were no significant differences between normal and cancerous tissue in the expression of the PD-1 and $P D-L 1$ genes $(p=0.724$ and $p=0.102$, respectively). However, $P D-1$ and $P D-L 1$ were both more highly expressed in high-grade bladder cancer than in low-grade bladder cancer $(p<0.050$ and $p<0.010)$. PD-L1 was positively correlated with the expressions of both the STAT1 $(r=0.681, p<0.001)$ and the NFATC1 genes $(r=0.444 . p<0.001)$.

Conclusions: $P D-1$ and PD-L1 might be a new biomarker that correlates with the pathological grade of bladder cancer. PD-L1 might function as a mediator of stage progression in bladder cancer and STAT1-NFAT pathway might associate this function.
\end{abstract}

Keywords: Programmed cell death protein 1, Programmed death-ligand 1, B7-H1, CD274, STAT1, NFATc1

\section{Background}

Bladder cancer has been characterized as a tumor group in which the immunological response is relatively well preserved [1-4]. Programmed death ligand 1 (PD-L1, B7-H1, CD274) is expressed in several malignancies, including bladder cancer [5-7]. The clinicopathological impact of this biomarker has not

\footnotetext{
* Correspondence: nakaigan@yokohama-cu.ac.jp

${ }^{1}$ Department of Urology, Yokohama City University Graduate School of Medicine, 3-9 Fukuura, Kanazawa-ku, Yokohama, Kanagawa 2360004, Japan Full list of author information is available at the end of the article
}

been established across different tumor types [8]. The programed death-1 (PD-1)/PD-L1 pathway negatively regulates $\mathrm{T}$ cell activation and has been suggested to play an important role in regulating host antitumor immunity [1].

Recently, several clinical trials targeting PD-1/PD-L1 pathway using anti-PD-1 antibody or anti-PD-L1 antibody demonstrated the obvious benefit for the patients with urothelial cancer and were approved by Food and Drug Administration in the United States [9-11]. Interestingly, the anti-tumor effect of atezolizumab, 
the anti-PD-L1 antibody, was dependent on the PD-L1 expression status on tumor-infiltrating immune cells [9]. On the other hand, the anti-tumor effect of pembrolizumab, an anti-PD-1 antibody, was not affected by the PD-L1 expression in the tumor and infiltrating immune cells [10], nor was the anti-tumor effect of nivolumab (also an anti-PD-1 antibody) affected by the PD-L1 expression in tumor cells [11]. In addition, some investigators reported that bladder cancer expressing high PD-L1 showed a poor prognosis [1, 12, 13], but others suggested high PD-L1 predicted the good prognosis [14]. Thus, the correlation between $P D-L 1$ expression and the prognosis remains controversial.

The upregulation of $P D-L 1$ expression by tumor cells, however, is thought to be a mechanism by which solid tumors develop a tolerance to immune regulation [15]; however, the detailed mechanism of PD-L1 in urothelial carcinoma remains unknown.

We previously reported the anti-cancer progression role in signal transducer and activator of transcription 1 (STAT1) and nuclear factor of activated T-cells (NFAT) [16-18]. This study evaluated the expressions of both $P D-1$ and $P D-L 1$ in urothelial carcinoma and firstly examined the PD-L1 related genes: STAT1 and NFAT.

\section{Methods}

\section{Patients and tissue specimens}

To analyze gene expression, we retrieved 58 pairs (116 specimens) of bladder tissue specimens that were obtained via transurethral resection of the bladder at Yokohama City University Hospital (Yokohama, Japan) and frozen immediately after resection. Appropriate approval was obtained from the institutional review board at our institution. The median age of the patients at resection was 72 years (mean age: $70.2 \pm$ 12.6 years). All patients were histologically diagnosed with bladder cancer, including $22(37.9 \%)$ patients who were diagnosed with low-grade bladder cancer and 21 (36.2\%) patients who were diagnosed with high-grade bladder cancer (Table 1). Both cancerous and non-cancerous tissue was pathologically obtained, and the non-cancerous tissue was obtained at least $1 \mathrm{~cm}$ away from the tumor site. The median follow-up period was 2.8 years. None of the patients received any pre-operative therapies, including BCG, radiation, or other anticancer drugs.

\section{Cell lines and Western blotting}

Human urothelial carcinoma cell lines (UMUC3, TCC-SUP HTB-3, T24, and 5637) obtained from the American Type Culture Collection (Manassas, VA, USA) were maintained in Dulbecco's modified Eagle's medium (DMEM; Mediatech, Manassas, VA, USA)
Table 1 Patients' Background

\begin{tabular}{ll}
\hline & Median (mean \pm SD) or $n(\%)$ \\
\hline No. of Pts. & 58 \\
Age $(y r)$ & $72(70.2 \pm 12.6)$ \\
Male / Female & $10(17.2 \%) / 48(82.8 \%)$ \\
Pathological Grade & \\
$\quad$ Low & $22(37.9 \%)$ \\
$\quad$ High & $21(36.2 \%)$ \\
unknown / other & $15(25.9 \%)$ \\
Pathological Stage & \\
$\quad$ Ta & $23(39.7 \%)$ \\
$\quad>T 1$ & $19(32.8 \%)$ \\
$\quad$ unknown & $16(27.6 \%)$ \\
Death $(n, \%)$ & $5(6.9 \%)$ \\
\hline
\end{tabular}

supplemented containing 10\% fetal bovine serum (FBS) with penicillin (100 units $/ \mathrm{mL})$ and streptomycin (100 units $/ \mathrm{mL}$ ) at $37{ }^{\circ} \mathrm{C}$ in a humidified atmosphere of $5 \% \mathrm{CO}_{2}$. Protein extraction and Western blotting were performed as described previously with minor modifications. In brief, equal amounts of protein $(30-50 \mu \mathrm{g})$ obtained from cell extracts were harvested for a total protein analysis. Extracted protein was separated using $10 \%$ sodium dodecylsulfate (SDS)-polyacrylamide gel electrophoresis (PAGE) and transferred to a polyvinylidene difluoride membrane (Immun-Blot PVDF Membrane; BIO-RAD, Hercules, CA, USA) by electroblotting using a standard protocol.

\section{Quantitative real-time RT-PCR}

Total RNA $(0.5 \mu \mathrm{g})$, which was isolated from the bladder tissue specimens, using Isogen (NipponGene, Tokyo, Japan), was reverse transcribed using $1 \mu \mathrm{M}$ oligo (dT) primers (Qiagen, Germantown, MD, USA) and 4 units of Omniscript reverse transcriptase (Qiagen, Germantown, MD, USA) in a total volume of $20 \mu \mathrm{L}$. Real-time quantitative PCR (qPCR) was then performed (StepOne Real Time PCR System, Applied Biosystems, Grand Island, NY, USA), using Fast SYBR Green Mastermix (Applied Biosystems, Grand Island, NY, USA), as described previously (22086872) [19] The following primer pairs were used for the RT-PCR: human PD-L1: forward 5' - CCA AGG CGC AGA TCA AAG AGA'; reverse 5' - AGG ACC CAG ACT AGC AGC A -3', and human NFATc1: forward 5'- GTC CCA CCA CCG AGC CCA CTA CG -3'; reverse 5' GAC CAT CTT CTT CCC GCCC ACG AC -3'. Human GAPDH: forward 5'-CTC CTC CAC CTT TGA CGC TG-3'; reverse, 5'-CAT ACC AGG AAA TGA GCT TGA CAA-3' was used as an internal control. The sequences of these primers were acquired from the Primer Bank: 19906719 [20], 19,108,745 [21], 
14,654,707 [22]. The PD-1 and STAT1 gene expressions were determined using TaqMan ${ }^{\circ}$ Gene Expression Assays (PD-L1 and STAT1, Applied Biosystems, Grand Island, NY, USA). All of the specific expression levels were divided by the quantity of GAPDH that was used.

\section{Statistical analyses}

The patients' characteristics were analyzed by the Mann-Whitney $U$ and chi-square tests. Continuous variables are expressed as the median and as the mean $( \pm \mathrm{SD})$. The patients' survival rates were calculated by the Kaplan-Meier method and comparisons were made by a $\log$-rank test. A $P$ value of $<0.05$ was considered to indicate statistical significance. A statistical analysis was performed and figures were created using the Graph Pad Prism software program (Graph Pad Software, La Jolla, CA, USA).

\section{Results}

\section{PD-L1 expression was observed in bladder cancer cell} lines

$P D-L 1$ was expressed in all human bladder cancer cell lines according to Western blotting (Additional file 1: Figure S1).

PD-1 and PD-L1 are more highly expressed in high-grade bladder cancer than in low-grade bladder cancer

$P D-1$ and $P D-L 1$ were expressed in human bladder cancer tissues. There were no differences between normal and cancerous bladder tissue both $P D-1$ and $P D-L 1$ gene expression ( $p=0.724, p=0.102$, respectively) (Fig. 1). Both $P D-1$ and $P D-L 1$ showed higher expression in high-grade tumors than in low-grade tumors $(p<0.050$, $p<0.010$, respectively) (Fig. 2). No correlation was found with the overall survival or pathological T stage.

\section{PD-L1 expression was correlated with STAT1 and NFATc1 expression}

To compare the expression of the $P D-L 1$ gene, we analyzed the expressions of the STAT1 and NFATC1 genes in addition to the expression of $P D-1$. The expressions of $P D-1$ and $P D-L 1$ were positively correlated, but not strongly $(r=0.224, p<0.05)$. PD-L1 was also positively correlated with STAT1 $(r=0.681, \quad p<0.001)$ and NFATc1 $(r=0.444 . p<0.001)$ (Fig. 3).

\section{Discussion}

This study demonstrated that $P D-L 1$ was more highly expressed in high-risk tumors than in low-risk ones. Urinary bladder cancer is the fourth-most commonly diagnosed malignancy in men in the United States, accounting for $6.7 \%$ of all cancer cases [23]. Two-thirds to three-quarters of patients with bladder cancer are initially diagnosed as non-muscle-invasive tumors. The concept for treatment of non-muscle-invasive tumors is conservative. But some cases progress to muscle-invasive tumors after recurrence, which have a risk of metastasis and threating life. However, current molecular markers remain insufficient to predict the potential for tumor recurrence and progression precisely. PD-L1 expression is present on antigen-presenting cells (APCs), such as human monocytes, as well as activated human and murine dendritic cells [24]. PD-L1 is a corregulatory ligand that can inhibit immune responses by either binding to $\mathrm{PD}-1$ or a putative non-PD-1 receptor on the surface of $\mathrm{T}$ lymphocytes to induce antigen-specific T-cell apoptosis or anergy [24]. The role of PD-L1 was evaluated as a mechanism for local stage progression in cancer [24]. PD-L1 might act as a mediator of stage progression in bladder cancer. On the other hand, the association between PD-1/ PD-L1 expression and progression is remains controversial. The correlation between PD-L1 expression in tumor cells and a worse clinical outcome was first reported in a study of 65 patients with bladder cancer by Nakanishi et al. [1]. Sharma et al. showed that the presence of PD-L1 tumor cells was not a predictor of prognosis [11]. Most reports have shown an association between the higher expression of PD-1/PD-L1 and a worse prognosis in bladder cancer.
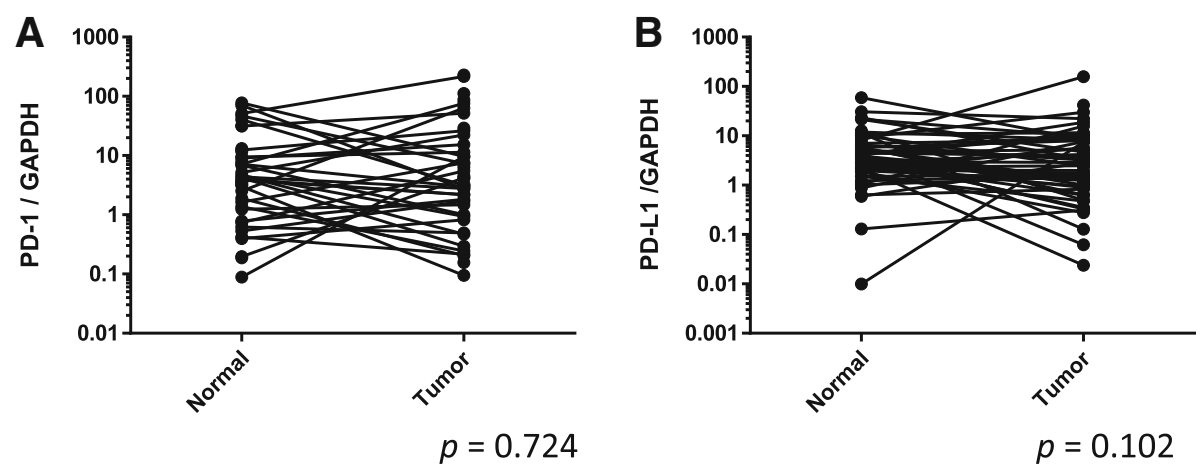

Fig. 1 PD-1 and PD-L1 gene expression in paired normal and tumor tissue specimens 

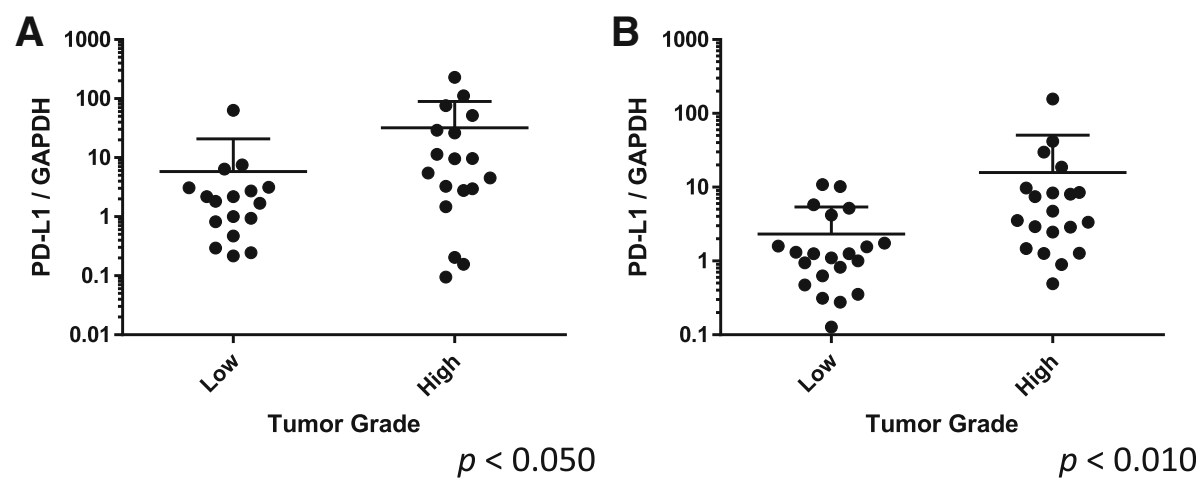

Fig. 2 PD-1 and PD-L1 gene expression with consideration of pathological grade

Previous studies have implicated several cytokines, including IFN , TNF $\alpha$, and IL-2, as possible regulators of PD-L1 expression on the surface of several tumor cells [25-27], and IFN has been thought as a strong inducer of PD-L1 expression in cancer cells [28]. Our study revealed that the expressions of STAT-1 and NFATc1 were positively correlated with PD-L1 expression. STAT-1 is downstream of IFNr and upstream of PD-L1 [29]. Following this cascade, NFAT is activated by PD-L1 [30]. We previously reported the anti-tumorigenic and oncogenic activity of NFAT in urothelial carcinoma and noted, in particular, that NFATc1 has a key role in its progression [16-18]. This correlation might therefore be a clue to reveal the anti-tumorigenic activity of PD-L1 through the STAT1-PD-L1-NFATc1 pathway.
The present study is associated with several limitations. First, this study was a retrospective analysis involving relatively few patients. Furthermore, all of the bladder cancer and paired normal tissue specimens were obtained via transurethral resection of the bladder, resulting in the potential for selection bias. Second, although the expression of $P D-1$ and $P D-L 1$ differed between high-grade and low-grade cancers, we did not assess the therapeutic outcome or detailed mechanisms underlying the development of bladder cancer. Most studies involving PD-1 and PD-L1 antibodies are performed in the advanced stages of cancer and not as a primary therapy. Thus, further studies are needed, including central pathologists diagnosis. However, this is the first report in a Japanese cohort, which represents a strength of this study.
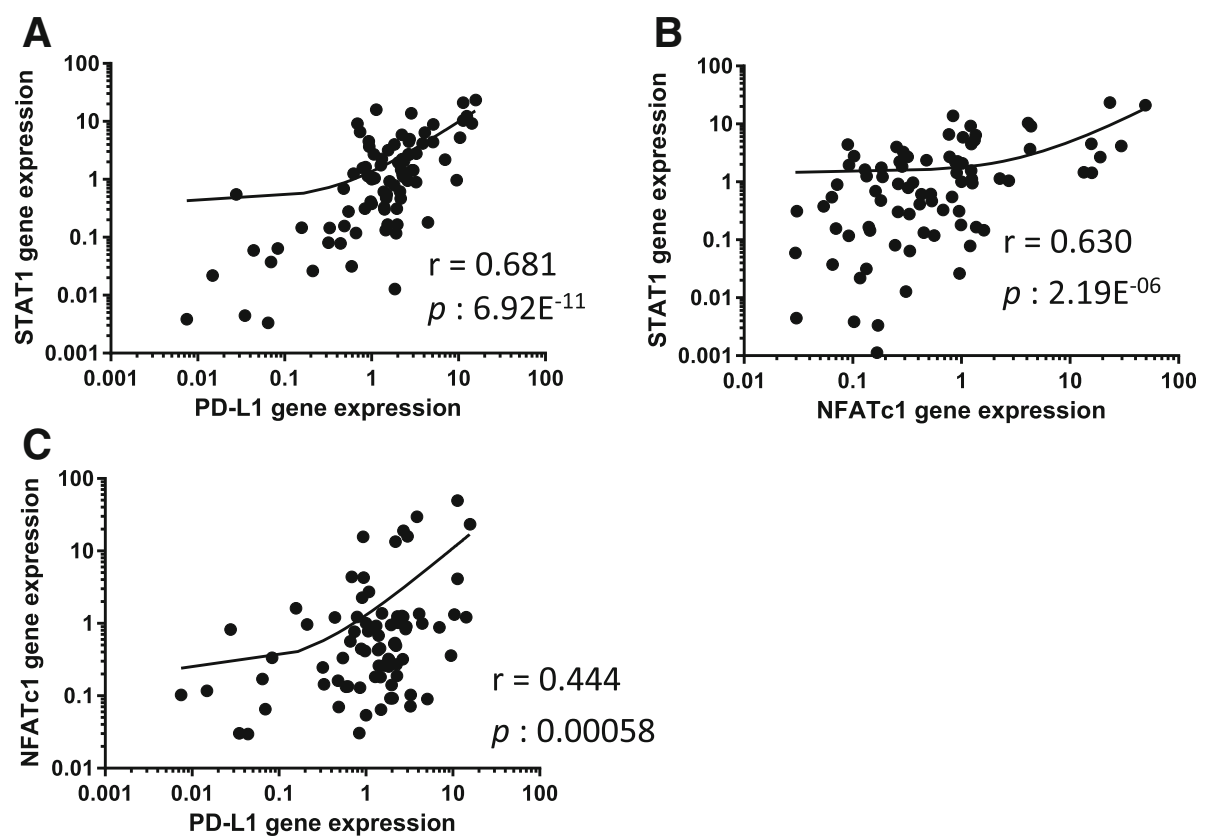

Fig. 3 The correlations among PD-L1, STAT1, and NFATC1 gene expression 


\section{Conclusions}

$P D-1$ and $P D-L 1$ might be new biomarkers that are correlated with the pathological grade of bladder cancer. $P D-L 1$ might act as a potential mediator of stage progression in bladder cancer. STAT1-NFAT pathway might associate this role.

\section{Additional file}

Additional file 1: Figure S1. The expression of $P D-L 1$ in human bladder cancer cell lines. (PPTX $71 \mathrm{~kb}$ )

\section{Abbreviations}

NFAT: Nuclear factor of activated T-cells; PD-1: Programmed death 1; PDL1: Programmed death ligand 1; qPCR: Quantitative real-time polymerase chain reaction; STAT 1: Signal transducer and activator of transcription 1

\section{Acknowledgements}

Not applicable.

\section{Funding}

Grants from KAKENHI grants (16 K20152) from the Ministry of Education, Culture, Sports, Science and Technology of Japan

\section{Availability of data and materials}

Due to ethical restrictions, the raw data that was used in this study is available upon request from the corresponding author.

\section{Authors' contributions}

Conceived and designed the experiments: TK, NN. Analyzed data: TK, NN. Performed the experiments: TK, SO, IK, Ylshiguro, HI, KMakiyama, HU, MY, NN. Acquisition of data: Ylto, KK, YM, YY, NH, HH, KO, KMuraoka, Kl, JT. Wrote the paper: TK, NN. All authors read and approved the final manuscript.

\section{Ethics approval and consent to participate}

Ethnic approval was obtained from the Ethics Committee of Yokohama City University Medical Center. Written informed consent was obtained from all patients for enrollment in this study.

\section{Consent for publication}

Written informed consent was obtained from all patients for publication of accompanying clinical records.

\section{Competing interests}

The authors declare that there is no conflict of interest regarding the publication of this paper.

\section{Publisher's Note}

Springer Nature remains neutral with regard to jurisdictional claims in published maps and institutional affiliations.

\section{Author details \\ 'Department of Urology, Yokohama City University Graduate School of Medicine, 3-9 Fukuura, Kanazawa-ku, Yokohama, Kanagawa 2360004, Japan. 2Department of Pathology, Yokohama City University Hospital, Yokohama, Japan. ${ }^{3}$ Department of Urology and Renal Transplantation, Yokohama City University Medical Center, Yokohama, Japan.}

Received: 20 November 2017 Accepted: 25 October 2018 Published online: 06 November 2018

\section{References}

1. Nakanishi J, Wada Y, Matsumoto K, Azuma M, Kikuchi K, Ueda S. Overexpression of B7-H1 (PD-L1) significantly associates with tumor grade and postoperative prognosis in human urothelial cancers. Cancer Immunol Immunother. 2007;56(8):1173-82.
2. Tsujihashi $H$, Matsuda $H$, Uejima S, Akiyama $T$, Kurita $T$. Immunocompetence of tissue infiltrating lymphocytes in bladder tumors. J Urol. 1988;140(4):890-4.

3. Tsujihashi $H$, Matsuda $H$, Uejima S, Akiyama T, Kurita T. Immunoresponse of tissue infiltrating lymphocytes in bladder tumors. J Urol. 1989;141(6):1467-70.

4. Lipponen PK, Eskelinen MJ, Jauhiainen K, Harju E, Terho R. Tumour infiltrating lymphocytes as an independent prognostic factor in transitional cell bladder cancer. Eur J Can. 1992;29A(1):69-75.

5. Konishi J, Yamazaki K, Azuma M, Kinoshita I, Dosaka-Akita H, Nishimura M. B7-H1 expression on non-small cell lung cancer cells and its relationship with tumor-infiltrating lymphocytes and their PD-1 expression. Clin Cancer Res. 2004:10(15):5094-100.

6. Hamanishi J, Mandai M, Iwasaki M, Okazaki T, Tanaka Y, Yamaguchi K, Higuchi T, Yagi H, Takakura K, Minato N, et al. Programmed cell death 1 ligand 1 and tumor-infiltrating CD8+ T lymphocytes are prognostic factors of human ovarian cancer. Proc Natl Acad Sci U S A. 2007;104(9):3360-5.

7. Inman BA, Sebo TJ, Frigola X, Dong H, Bergstralh EJ, Frank I, Fradet $Y$, Lacombe L, Kwon ED. PD-L1 (B7-H1) expression by urothelial carcinoma of the bladder and BCG-induced granulomata: associations with localized stage progression. Cancer. 2007;109(8):1499-505.

8. Bellmunt J, Orsola A, Sonpavde G. Precision and predictive medicine in urothelial cancer: are we making progress? Eur Urol. 2015 Oct;68(4):547-9.

9. Rosenberg JE, Hoffman-Censits J, Powles T, et al. Atezolizumab in patients with locally advanced and metastatic urothelial carcinoma who have progressed following treatment with platinum-based chemotherapy: a single-arm, multicenter, phase 2 trial. Lancet. 2016;387:1909-20.

10. Bellmunt J, de Wit R, Vaughn DJ, et al. Pembrolizumab as second-line therapy for advanced urothelial carcinoma. N Engl J Med. 2017:376:1015-26.

11. Sharma P, Retz M, Siefker-Radtke A, et al. Nivolumab in metastatic urothelial carcinoma after platinum therapy (CheckMate 275): a multicentre, singlearm, phase 2 trial. Lancet Oncol. 2017:18:312-22.

12. Xylinas $E$, Robinson $B D$, Kluth $L A$, et al. Association of T-cell co-regulatory protein expression with clinical outcomes following radical cystectomy for urothelial carcinoma of the bladder. Eur J Surg Oncol. 2014;40:121-7.

13. Boorjian SA, Sheinin Y, Crispen PL, Farmer SA, Lohse CM, Kuntz SM, Leibovich BC, Kwon ED, Frank I. T-cell coregulatory molecule expression in urothelial cell carcinoma: clinicopathologic correlations and association with survival. Clin Cancer Res. 2008;14(15):4800-8.

14. Breyer J, Wirtz RM, Otto W, et al. High PDL1 mRNA expression predicts better survival of stage pT1 non-muscle-invasive bladder cancer (NMIBC) patients. Cancer Immunol Immunother. 2017. https://doi.org/10.1007/ s00262-017-2093-9 [Epub ahead of print].

15. Hafez N, Petrylak DP. Could PD-L1 prove to be an effective therapeutic target for bladder cancer? Immunotherapy. 2015;7(1):1-2.

16. Kawahara T, Kashiwagi E, Ide H, Li Y, Zheng Y, Ishiguro H, Miyamoto H. The role of NFATc1 in prostate cancer progression: cyclosporine a and tacrolimus inhibit cell proliferation, migration, and invasion. Prostate. 2015;75(6):573-84.

17. Kawahara T, Kashiwagi E, Ide H, Li Y, Zheng Y, Miyamoto Y, Netto GJ, Ishiguro H, Miyamoto H, Cyclosporine A. Tacrolimus inhibit bladder cancer growth through down-regulation of NFATc1. Oncotarget. 2015:6(3):1582-93.

18. Kawahara T, Kashiwagi E, Li Y, Zheng Y, Miyamoto Y, Netto GJ, Ishiguro H, Miyamoto $\mathrm{H}$. Cyclosporine A and tacrolimus inhibit urothelial tumorigenesis. Mol Carcinog. 2016;55(2):161-9.

19. Izumi K, Zheng Y, Hsu JW, Chang C, Miyamoto H. Androgen receptor signals regulate UDP-glucuronosyltransferases in the urinary bladder: a potential mechanism of androgen-induced bladder carcinogenesis. Mol Carcinog. 2013;52(2):94-102.

20. Spandidos A, Wang $X$, Wang $H$, Seed B. PrimerBank: a resource of human and mouse PCR primer pairs for gene expression detection and quantification. Nucleic Acids Res. 2010;38(Database issue):D792-9.

21. Spandidos A, Wang X, Wang H, Dragnev S, Thurber T, Seed B. A comprehensive collection of experimentally validated primers for polymerase chain reaction quantitation of murine transcript abundance. BMC Genomics. 2008;9:633.

22. Wang $X$, Seed B. A PCR primer bank for quantitative gene expression analysis. Nucleic Acids Res. 2003;31(24):e154

23. Jemal A, Siegel R, Xu J, Ward E. Cancer statistics, 2010. CA Cancer J Clin. 2010;60(5):277-300.

24. Pardoll DM. The blockade of immune checkpoints in cancer immunotherapy. Nat Rev Cancer. 2012;12(4):252-64. 
25. Dong H, Strome SE, Salomao DR, Tamura H, Hirano F, Flies DB, Roche PC, Lu J, Zhu G, Tamada K, et al. Tumor-associated B7-H1 promotes Tcell apoptosis: a potential mechanism of immune evasion. Nat Med. 2002;8(8):793-800

26. Youngnak-Piboonratanakit $P$, Tsushima F, Otsuki N, Igarashi H, Machida U, Iwai H, Takahashi Y, Omura K, Yokozeki H, Azuma M. The expression of B7$\mathrm{H1}$ on keratinocytes in chronic inflammatory mucocutaneous disease and its regulatory role. Immunol Lett. 2004;94(3):215-22.

27. Wintterle S, Schreiner B, Mitsdoerffer M, Schneider D, Chen L, Meyermann $R$, Weller $M$, Wiendl $H$. Expression of the B7-related molecule B7-H1 by glioma cells: a potential mechanism of immune paralysis. Cancer Res. 2003;63(21):7462-7.

28. Topalian SL, Taube JM, Anders RA, Pardoll DM. Mechanism-driven biomarkers to guide immune checkpoint blockade in cancer therapy. Nat Rev Cancer. 2016;16(5):275-87.

29. Kharma B, Baba T, Matsumura N, Kang HS, Hamanishi J, Murakami R, McConechy MM, Leung S, Yamaguchi K, Hosoe Y, et al. STAT1 drives tumor progression in serous papillary endometrial cancer. Cancer Res. 2014;74(22): 6519-30.

30. Chunyang LXX. Hongyan Wang, bin Wei: PD-1 and CTLA-4 mediated inhibitory signaling for T cell exhaustion during chronic viral infections. J Clin Cell Immunol. 2012:S12:010.

Ready to submit your research? Choose BMC and benefit from:

- fast, convenient online submission

- thorough peer review by experienced researchers in your field

- rapid publication on acceptance

- support for research data, including large and complex data types

- gold Open Access which fosters wider collaboration and increased citations

- maximum visibility for your research: over $100 \mathrm{M}$ website views per year

At BMC, research is always in progress.

Learn more biomedcentral.com/submissions 